\title{
Metastatic gastric cancer from breast carcinoma: A report of 78 cases
}

\author{
LIANG XU ${ }^{1,2}$, SHUJING LIANG ${ }^{1,2}$, NINGNING YAN ${ }^{1,2}$, LE ZHANG $^{1,2}$, HAILIANG GU $^{3}$, \\ XIAOCHUN FEI ${ }^{4}$, YINGCHUN XU ${ }^{5}$ and FENGCHUN ZHANG ${ }^{1,2}$
}

\author{
${ }^{1}$ Department of Oncology, Suzhou Kowloon Hospital, Shanghai Jiaotong University School of Medicine, Suzhou, \\ Jiangsu 215021; ${ }^{2}$ Department of Oncology, Ruijin Hospital, Shanghai Jiaotong University School of Medicine, Shanghai \\ 200025; ${ }^{3}$ Department of General Surgery, The First Affiliated Hospital of Soochow University, Suzhou, \\ Jiangsu 215006; Departments of ${ }^{4}$ Pathology and ${ }^{5}$ Oncology, Renji Hospital, \\ Shanghai Jiaotong University School of Medicine, Shanghai 200127, P.R. China
}

Received November 22, 2015; Accepted December 16, 2016

DOI: $10.3892 / 01.2017 .6703$

\begin{abstract}
The metastatic spread of breast carcinoma to the stomach is rare. There are a small number of previous studies that report metastases from the breast to the stomach and these provide limited information regarding this infrequent event. Consequently, the clinicopathological features, clinical outcomes and the optimal treatment for these patients remain to be elucidated. In the present study, 78 cases of gastric metastases from breast cancer, including the current case, were identified from previous studies between 1960 and 2015 . The clinicopathological features of primary breast tumors and metastatic gastric lesions, including initial stage, tumor size, hormone receptor status, treatment modalities and overall survival (OS) rate, were analyzed. The patients were all female and the median age at the time of gastric metastasis diagnosis was 59 years old (range, 38-86 years). The majority of the patients initially presented with stage II breast cancer (35.9\%) and abdominal pain was the most common symptom of gastric metastases (75.6\%). A total of 51/78 patients (65.4\%) were identified to have a history of invasive lobular breast carcinoma and the majority of gastric tumors were positive for hormonal receptors and human epidermal growth factor receptor 2 (HER-2) negative (estrogen receptor, 94.0\%; progesterone receptor, $68.3 \%$; HER-2, 5.9\%). Furthermore, in the univariate analysis, multiple organs involved prior to or at the
\end{abstract}

Correspondence to: Professor Yingchun $\mathrm{Xu}$, Department of Oncology, Renji Hospital, Shanghai Jiaotong University School of Medicine, 1630 Dongfang Road, Shanghai 200127, P.R. China E-mail: namex1l@163.com

Professor Fengchun Zhang, Department of Oncology, Suzhou Kowloon Hospital, Shanghai Jiaotong University School of Medicine, 118 Wansheng Street, Suzhou, Jiangsu 215021, P.R. China E-mail: fczhang2005@163.com

Key words: breast cancer, gastric metastasis, metastatic tumor, invasive lobular carcinoma, hormone therapy time of gastric metastases were diagnosed and multiple gastric lesions and peritoneal carcinomatosis were significantly correlated with OS. Additionally, salvage hormonal therapy, but not surgery or chemotherapy, significantly extended OS. However, in the multivariate analysis, metastasis prior to stomach involvement was the only independent indicator of poor OS. In conclusion, physicians must be vigilant when patients with breast cancer history present with gastrointestinal symptoms, despite gastric metastasis from breast cancer being rare. An appropriate systemic therapeutic strategy that includes hormonal therapy may be beneficial for this group of patients.

\section{Introduction}

Breast cancer is established as a global public health concern and it is predicted to account for $29 \%$ of newly diagnosed cancer cases in women (1). Although there has been a gradual improvement in treatment for patients with breast cancer, a number of these patients may develop distant metastases. Breast cancer frequently metastasizes to the local and distant nodes, lungs, bones, liver or brain (1). However, it is rare for breast cancer to metastasize to the gastrointestinal system, particularly to the stomach (2). The estimated rate of breast to stomach metastasis varies from $0.3 \%$ in retrospective series to $8-18 \%$ in autopsy series (3). Due to the low incidence, only sporadic cases or small series of gastric metastases from breast cancer have currently been identified. Therefore, little is established on the clinicopathological characteristics, clinical outcomes, endoscopic features and, particularly, the prognostic factors and appropriate treatments for these patients.

Therefore, the current study investigated a primary breast cancer metastasized to the stomach, which was diagnosed using gastroscopy and immunohistochemistry analysis. Additionally, the present study reviewed 77 similar cases that have been reported in previously published studies. The aim of the current study was to perform a retrospective analysis of a cohort of patients with breast cancer and gastric metastases to examine the clinicopathological and endoscopic features, the treatment modalities and the factors associated with prolonged survival. In the present study, it was revealed that gastric 
metastasis from breast cancer is more common in invasive lobular breast cancer compared with infiltrative ductal breast cancer. In addition, abdominal pain was revealed to be the most common symptom. Therefore, when a stomach mass is detected in a patient with lobular breast cancer, it may be either a primary stomach cancer or the metastasis of the breast cancer, although the gastric metastasis from breast cancer is quite rare. Additionally, histological and immunopathological analysis may aid the differential diagnoses. For the gastric metastasis from breast cancer with hormone receptor overexpression (such as estrogen receptor and progesterone receptor), systemic treatment strategy, including hormonal therapy, is also recommended.

\section{Materials and methods}

Case report. A 73-year-old female was admitted to the Department of Oncology, Renji Hospital, Shanghai Jiaotong University School of Medicine (Shanghai, China), who presented with anorexia, fatigue, abdominal distention and a history of multiple skin nodules in the chest wall for the prior two months. On physical examination, the chest wall nodules appeared as solid, irregular and fixed, measuring $0.5-1.2 \mathrm{~cm}$ in diameter. A core needle biopsy was performed and histopathological studies identified the infiltration of malignant tumor cells into the dermis. Additionally immunohistochemistry analysis identified that the tumor cells were positive for the estrogen receptor (ER) and the progesterone receptor (PR), but negative for epithelial-cadherin (E-CAD) and cluster of differentiation (CD) 20 (Fig. 1A) indicating the immunophenotype was consistent with lobular carcinoma of the breast.

The patient also identified that $\sim 1$ year prior to the onset of gastrointestinal symptoms, they identified the painless bilateral breast masses but did not present to a clinician. Subsequently, the patient underwent a bilateral breast magnetic resonance imaging examination, which identified bilateral mammary gland hyperplasia and diffuse patchy shadows with nodular enhancement determined to be category 3 on the Breast Imaging Reporting and Data System mammographic assessment scale (4). Bilateral breast core biopsies were performed and the histopathology examination identified invasive lobular carcinoma (ILC) with positive ER and PR staining (Fig. 1B). In addition, immunohistochemical stains also exhibited negative HER-2 and weakly positive ki-67 (5\%).

The following staging examinations, including chest and whole brain computed tomography (CT), abdominal ultrasound scan and bone emission CT identified synchronous metastases in bones, lungs, pleura and the stomach. The CT scan of the chest of the patient identified multiple pulmonary nodules and pleural effusion of the two sides of thorax with a possible lymphangitic metastasis. Multiple hyperdense lesions in the thoracic vertebrae were identified. An abdominal CT scan revealed a gastric wall thickening with characteristics of linitis plastic; however, there was no evidence of intra-abdominal spread (Fig. 2A). Similarly, gastroscopy evaluation identified rigid gastric folds with poor distensibility due to the presence of diffuse infiltrative lesions that involved the entire stomach (Fig. 2B). No evidence of Helicobacter pylori infection was noted. Pathological analysis of the endoscopic biopsy samples demonstrated diffuse infiltration with poorly differentiated adenocarcinoma. Immunohistochemistry analysis identified that the tumor cells were positive for ER, PR, gross cystic disease fluid protein (GCDFP) -15 and cytokeratin (CK) 7 but not for CK20 (Fig. 3) supporting the diagnosis of a metastatic gastric cancer from the breast carcinoma. A tumor-marker panel was analysed using a radioimmunoassay as previously described before (5), and the results indicated increased levels of cancer antigen (CA)153 (212.90 U/ml), CA125 (502.90 U/ml), CA199 (45.70 U/ml) and carcinoembryonic antigen (CEA; $21.62 \mathrm{ng} / \mathrm{ml})$. Therefore, the diagnosis was of the development of lung, bone and stomach metastases originating from lobular breast cancer.

Subsequently, zoledronic acid (4 mg every 4 weeks) was administrated to the patient for the treatment of metastatic bone disease and to prevent skeletal-related events. Simultaneously, hormonal therapy (letrozole, $2.5 \mathrm{mg} / \mathrm{day}$ ) was also administered. After nine months, the bilateral breast masses and the chest wall nodules had reduced in volume significantly. The tumor lesions in the stomach and lungs were stable. Laboratory examinations indicated a decreased CA125 level to $107.4 \mathrm{U} / \mathrm{ml}$ and CA153 level to $60.57 \mathrm{U} / \mathrm{ml}$. The serum CEA returned to a normal level. At the 13-month follow-up, the patient's condition remained asymptomatic in March 2015 and the described treatment was continued.

Literature search for gastric metastases from breast cancer. A search of literature using the PubMed website of data published in English between January 1960 and October 2015 was performed with the key words 'stomach OR gastric', 'breast cancer' and 'metastasis OR metastases'. The reference lists of the original articles were also searched for relevant studies. The titles, abstracts and associated citations of the studies that were identified were reviewed. Only articles with available information were included in the analysis. The collected data included the epidemiological information, symptomatology, indications of endoscopic investigation, macroscopic presentation, time between primary breast tumor diagnosis and the detection of gastric metastasis, tumor treatment and prognosis.

Statistical analysis. Survival data was defined as the time from gastric metastasis until date of mortality or final follow-up and the median survival and overall survival rates were calculated. Univariate analyses were performed using the Kaplan-Meier method and groups were compared using a log-rank test. Multivariate analysis using the Cox proportional hazards model, including all factors with $\mathrm{P}<0.05$ from the univariate analysis, was performed to determine the impact of associated factors. All P-values were two-sided. All statistical analyses were performed using SPSS version 21.0 (IBM SPSS, Armonk, $\mathrm{NY}$, USA). $\mathrm{P}<0.05$ was considered to indicate a statistically significant difference.

\section{Results}

Clinicopathological characteristics of primary breast cancer. A total of 78 cases of primary breast cancer with gastric involvement were identified in studies obtained via PubMed. The characteristics of the primary breast cancer 
Table I. Clinicopathological information of primary breast tumor.

\begin{tabular}{lc}
\hline Variables & $\begin{array}{c}\text { Number of } \\
\text { patients }\end{array}$ \\
\hline
\end{tabular}

Age, years

$<40$

40-59

8

$\geq 60$

47

23

Median

53

Tumor position
Left

Right

Bilateral

Unknown

Tumor size

T0

T1

$\mathrm{T} 2$

T3

T4

Unknown

34

25

14

5

2

22

29

12

1

12

Lymph node involvement

Positive

Negative

Unknown

Stage $^{\text {a }}$

1

2

3

4

Unknown

Histology

ILC

IDC

Other

Unknown

ER status

Positive

Negative

Unknown

44

20

14

6

28

8

23

13

51

19

2

6

49

8

21

PR status

Positive

36

17

25

Unknown

HER-2 status

Positive

Negative

Unknown
10.3

60.3

29.5

43.6

32.1

17.9

6.4

2.6

28.2

37.2

15.4

1.3

15.4

56.4

25.6

17.9

7.7

35.9

10.3

29.5

16.7

65.4

24.4

2.6

7.7

62.8

10.3

26.9

46.1

21.8

32.1

6.4

51.3

42.3

ILC, invasive lobular cancer; IDC, invasive ductal cancer; ER, estrogen receptor; PR, progesterone receptor; HER-2, human epidermal growth

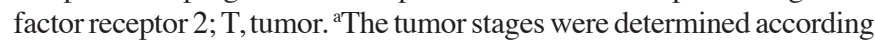
the 2003 American Joint Committee in Cancer staging manual.

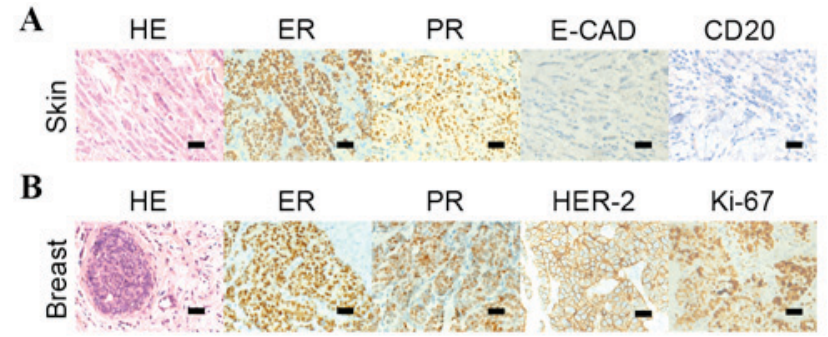

Figure 1. HE staining and immunohistochemistry analysis of the breast tumors, the skin nodules. (A) HE staining revealed the malignant cells in the skin nodules and the immunohistochemistry analysis indicated that the cells from the skin nodules were positive for ER and PR and negative for E-CAD and CD20. (B) HE staining identified the malignant cells of the primary breast tumor and the immunohistochemistry analysis detected that the cells from the breast tumor were positive for ER, PR, HER-2 and Ki-67.

cases identified from the literature search are summarized in Table I. The median age of primary breast cancer diagnosis was 53 years (range, 33-86; mean, 54.8) and all patients were female.

Tumor size was available in 66/78 cases, and the majority were identified as T1-stage (28.2\%) and T2-stage (37.2\%) breast cancer, according to the 2003 American Joint Committee on Cancer staging system (6). Two patients (2.6\%) presented with occult breast cancer. The majority of the patients initially presented with stage II cancer $(35.9 \%)$ and the remaining tumours were classified as follows: Stage I, 7.7\%; stage III, $10.3 \%$; stage IV, $29.5 \%$ (Table I). Stage information was unavailable in 13 patients $(16.7 \%)$ due to unknown tumor size or lymph node status. A total of 51 patients $(65.4 \%)$ had a history of breast ILC, whereas only 19 patients $(24.4 \%)$ were identified with a history of breast invasive ductal carcinoma (IDC). Additionally, lymph node involvement was detected in 44 patients $(56.4 \%)$, whereas 22 patients $(25.9 \%)$ had no nodal involvement. In the present study, ER and PR expression levels were positive in 49 cases $(62.8 \%)$ and $45(46.1 \%)$ cases, respectively. Human epidermal growth factor receptor-2 (HER-2) status was available in 67 cases and only 7 cases (6.4\%) were positive (Table I).

Clinicopathological characteristics of gastric metastasis. The clinicopathological characteristics of patients with gastric metastasis are presented in Table II. The median age at the gastric metastasis diagnosis was 59 years (range, 38-86; mean, 60.9). The median interval between the primary diagnosis and metastatic presentation was 60 months (range, 0-252; mean, 70.7). Gastric metastasis was identified at the same time as the breast cancer diagnosis in 18 patients (23.1\%). A total of 14 patients sought the initial consultation for gastrointestinal symptoms prior to the breast cancer being diagnosed (17.9\%).

A total of 27 patients were identified as having other organs that had already been affected when the diagnosis of gastric metastases was established (34.6\%). Furthermore, co-existing metastases in other tissues were present in $<62.8 \%$ of cases at the time of diagnosis of the stomach metastasis (Table II). The majority of additional metastases were located in the bone (50.0\%), colon (24.4\%), liver (20.4\%) and lung (12.2\%) (data not shown).

The majority of gastric metastases of breast cancer manifested as nonspecific symptoms, including dyspepsia, 
Table II. Log-rank analysis of clinical characteristics of gastric metastases with overall survival rate.

\begin{tabular}{|c|c|c|}
\hline Characteristics & $\begin{array}{l}\text { Number of } \\
\text { patients }(\%)\end{array}$ & P-value \\
\hline $\begin{array}{l}\text { Age at diagnosis of gastric } \\
\text { metastases, years }\end{array}$ & & 0.166 \\
\hline $0-49$ & $12(15.4)$ & \\
\hline$\geq 50$ & $66(84.6)$ & \\
\hline $\begin{array}{l}\text { Time between primary and } \\
\text { secondary cancers, years }\end{array}$ & & 0.998 \\
\hline $0-2$ & $28(35.9)$ & \\
\hline$\geq 2$ & $48(60.3)$ & \\
\hline Unknown & $2(2.6)$ & \\
\hline Location of lesion & & 0.160 \\
\hline Upper 3rd & $16(20.5)$ & \\
\hline Middle 3rd & $14(17.9)$ & \\
\hline Lower 3rd & $23(29.5)$ & \\
\hline Whole stomach & $18(23.1)$ & \\
\hline Unknown & $5(6.4)$ & \\
\hline Solitary lesion in stomach & & 0.025 \\
\hline Yes & $36(46.2)$ & \\
\hline No & $40(51.3)$ & \\
\hline Unknown & $2(2.6)$ & \\
\hline $\begin{array}{l}\text { Any other metastases prior } \\
\text { to stomach involved }\end{array}$ & & 0.006 \\
\hline Yes & $27(34.6)$ & \\
\hline No & $43(55.1 \%)$ & \\
\hline Unknown & $8(10.3)$ & \\
\hline $\begin{array}{l}\text { Any other metastases when } \\
\text { stomach involved }\end{array}$ & & 0.048 \\
\hline Yes & $49(62.8)$ & \\
\hline No & $29(37.2)$ & \\
\hline Peritoneal carcinomatosis & & 0.005 \\
\hline Yes & $36(46.2)$ & \\
\hline No & $41(52.7)$ & \\
\hline Unknown & $1(1.3)$ & \\
\hline Main symptom & & 0.765 \\
\hline Abdominal pain & $59(75.6)$ & \\
\hline Anorexia & $45(57.7)$ & \\
\hline Bleeding & $6(7.7)$ & \\
\hline Dysphagia & $4(5.1)$ & \\
\hline Vomiting & $15(19.2)$ & \\
\hline Asymptomatic & $5(6.4)$ & \\
\hline
\end{tabular}

Statistical analysis was univariate and performed using the log-rank test.

anorexia, bloating, melena, nausea, vomiting, early satiety and epigastric pain. Abdominal pain was the most frequent symptom in the current study $(75.6 \%)$. Endoscopy evaluation is essential in the diagnosis of gastric metastatic disease due to the diversity of clinical manifestations in the patients with
Table III. Treatment modalities and their association with overall survival rate in patients with breast cancer with stomach metastases.

\begin{tabular}{lcc}
\hline Characteristics & $\begin{array}{c}\text { Number of } \\
\text { patients }(\%)\end{array}$ & P-value \\
\hline Surgery & $32(41.0)$ & 0.134 \\
Yes & $42(53.8)$ & \\
No & $4(5.1)$ & \\
Unknown & & \\
Chemotherapy & $44(56.4)$ & \\
Yes & $28(35.9)$ & \\
No & $6(7.7)$ & \\
Unknown & & \\
Radiotherapy & $6(7.7)$ & \\
Yes & $68(87.2)$ & \\
No & $4(5.1)$ & \\
Unknown & & \\
Hormonal therapy & $40(51.3)$ & \\
Yes & $31(39.7)$ & \\
No & $5(6.4)$ & \\
Unknown &
\end{tabular}

Statistical analysis was univariate and performed using the log-rank test.

gastric metastases. The evaluation of endoscopic information identified 16 patients that had lesions in the upper third of the stomach $(20.5 \%), 14$ patients $(17.9 \%)$ that had lesions in the middle third of the stomach and 23 patients that had lesions in the lower third of the stomach (29.5\%; Table II). Solitary lesions in the stomach were identified in 36 cases (46.2\%) and 40 patients were identified to have multiple gastric metastatic lesions (51.3\%; Table II). Additionally, ER and PR status were able to be assessed in 50 and 41 cases, respectively, and the majority of gastric metastases were hormonal receptor positive (ER for $94.0 \%$ and PR for $68.3 \%$ ). However, in the 34 cases with HER-2 information, only $2 / 34$ cases were HER-2 positive (5.9\%; Table II).

Following the discovery of gastric metastatic disease, the majority of patients received salvage chemotherapy $(56.4 \%)$ or salvage hormonal therapy $(51.3 \%)$. Surgeries that were performed included total gastrectomy, subtotal gastrectomy and wedge resection, which were performed in a total of 32 patients $(41.0 \%)$. A small number of patients were treated with radiotherapy $(7.7 \%$; Table III).

Patient survival. The follow-up data was available for 74 patients. The median survival was 10.5 months (range, $0.25-116)$. The univariate analysis for the association between overall survival (OS) rate and clinicopathological and biological characteristics of the primary breast tumor and gastric metastasis was performed. According to the univariate analysis, multiple organ involvement prior to or at the time of gastric metastatic disease diagnosis and the presence of multiple gastric lesions, or peritoneal carcinomatosis were 
A

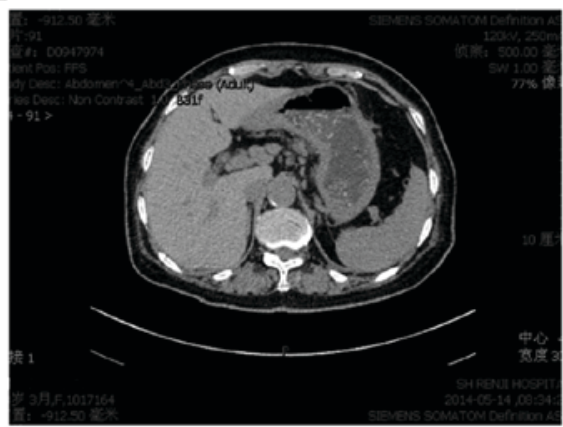

B

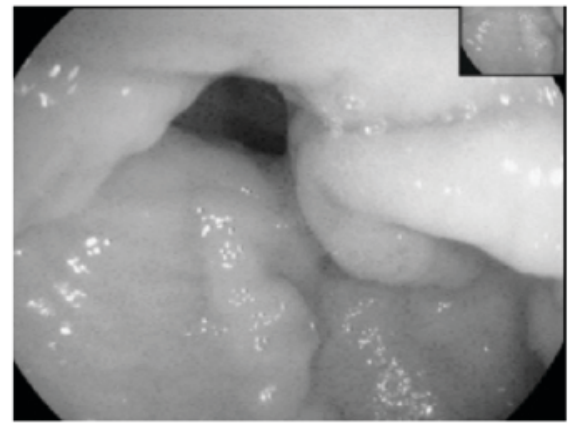

Figure 2. Computed tomography scan and gastroscopy examination of the gastric metastasis. (A) Abdominal tomography identified diffused thickening of gastric wall with a linitis plastica appearance. (B) Gastroscopy evaluation identified rigid gastric folds with decreased distensibility.

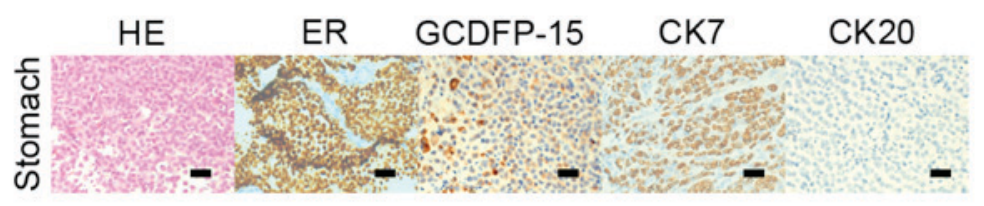

Figure 3. HE staining and immunohistochemistry analysis of the gastric metastases HE staining revealed the malignant cells in the gastric wall, and immunohistochemistry indicated that cells from the gastric tumor were positive for CK7, ER and GCDFP-15 and negative for CK20. Scale bar, $50 \mu \mathrm{m}$. HE, hematoxylin and eosin; ER, estrogen receptor, PR, progesterone receptor; E-CAD, epithelial cadherin; HER-2, human epidermal growth factor receptor 2; GCDFP-15, gross cystic disease fluid protein 15; CK7, cytokeratin 7; CK20, cytokeratin 20; CD20, cluster of differentiation 20.

A

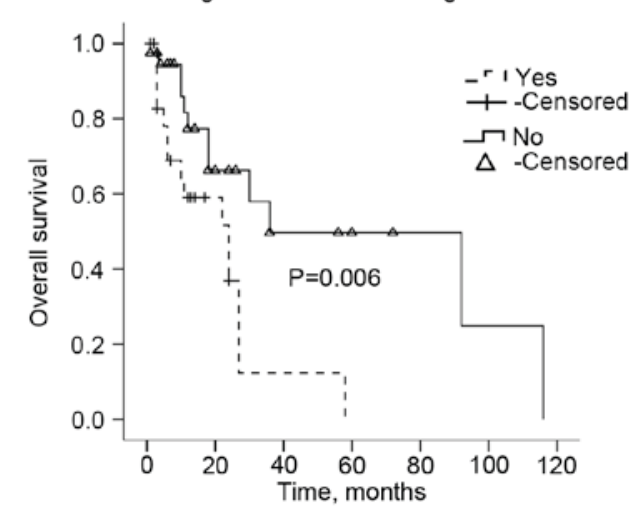

C

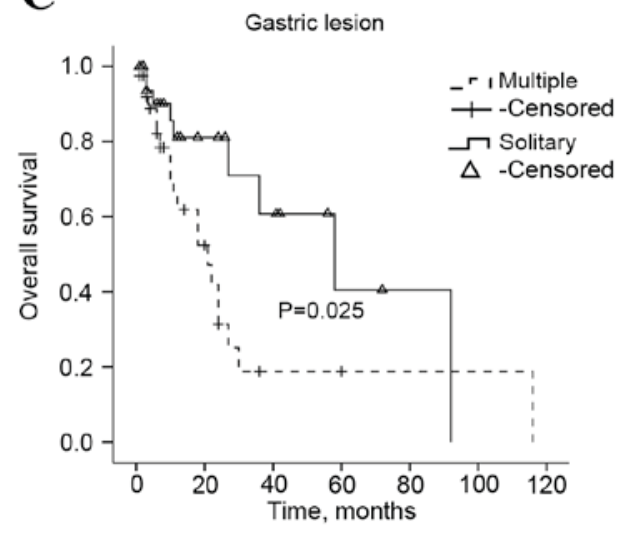

B

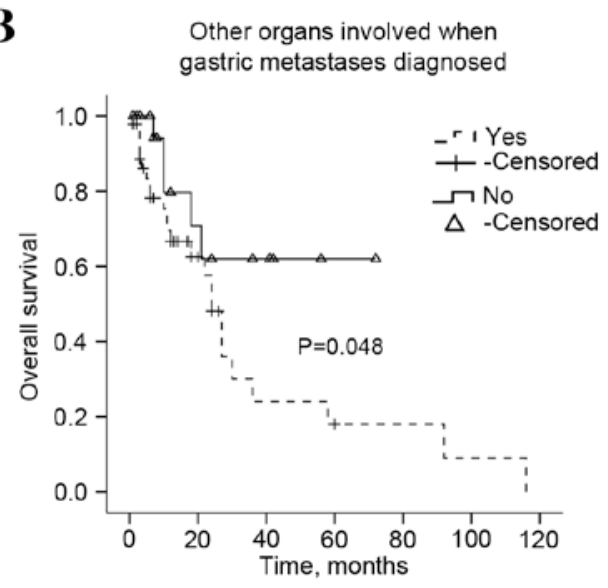

D

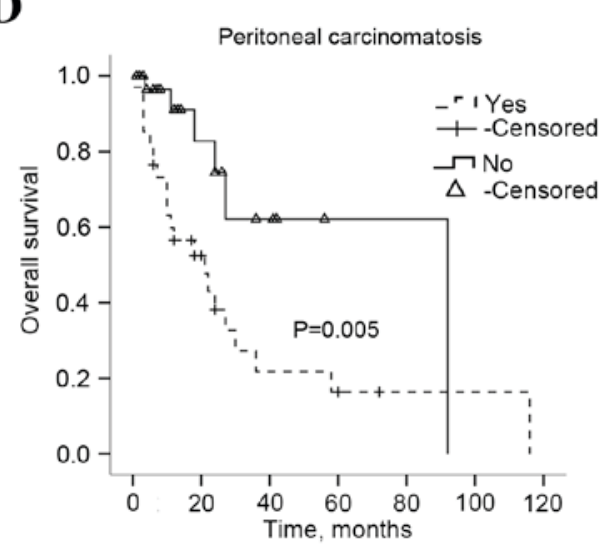

Figure 4. Overall survival rates of patients with gastric metastatic tumors with distinct clinical features. (A) Kaplan-Meier overall survival rate graph of the gastric metastases of patients with breast cancer and other organs involved prior to gastric metastases being diagnosed. (B) Kaplan-Meier overall survival rate graph of the gastric metastases of patients with breast cancer and with other organs involved when gastric metastases were diagnosed. (C) Kaplan-Meier overall survival rate graph of the gastric metastases of patients with breast cancer and with solitary or multiple gastric lesions. (D) Kaplan-Meier overall survival rate graph of the gastric metastases of patients with breast cancer and with peritoneal carcinomatosis. Statistical analysis was performed using the log-rank test. 
Table IV. Multivariate analysis of prognostic factors for OS in patients with gastric metastases.

\begin{tabular}{lcccccc}
\hline Variables & B & SE & Wald & P-value & OR & $95 \%$ CI \\
\hline Other metastases prior to stomach involvement & -1.127 & 0.445 & 6.414 & 0.011 & 0.324 & $0.135-0.775$ \\
Other metastases when stomach involved & -0.317 & 0.642 & 0.243 & 0.622 & 0.729 & $0.207-2.564$ \\
Salvage hormonal therapy & 0.749 & 0.442 & 2.864 & 0.091 & 2.114 & $0.888-5.030$ \\
Solitary lesion in stomach & 0.25 & 0.436 & 0.33 & 0.566 & 1.285 & $0.547-3.019$ \\
Peritoneal carcinomatosis & -0.717 & 0.484 & 2.192 & 0.139 & 0.488 & $0.189-1.261$ \\
\hline
\end{tabular}

B, $\beta$ test; SE, standard error; Wald, Wald $\chi^{2}$ test; OR, odds ratio; OS, overall survival rate; CI, confidence interval.
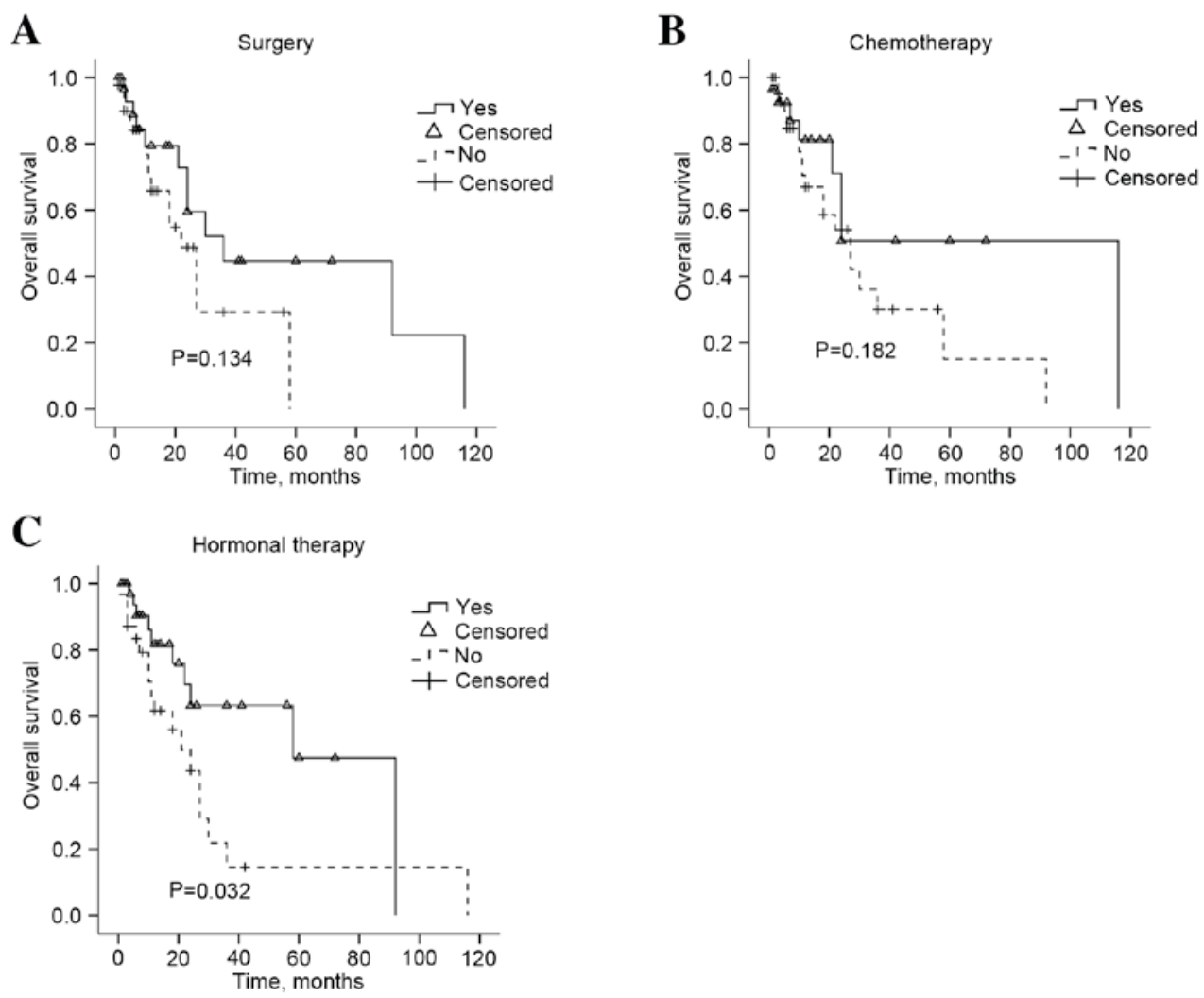

Figure 5. Overall survival rate in response to the treatment methods administered to patients with gastric metastases. Kaplan-Meier graph for the survival following (A) salvage surgery, (B) salvage chemotherapy and (C) salvage hormonal therapy. Statistical analysis was performed using the log-rank test.

significantly correlated with $\mathrm{OS}(\mathrm{P}=0.006, \mathrm{P}=0.048, \mathrm{P}=0.025$ and $\mathrm{P}=0.005$, respectively; Fig. 4). Treatment modalities were also investigated and salvage hormonal therapy $(\mathrm{P}=0.0320)$, but not surgery or chemotherapy $(\mathrm{P}=0.134, \mathrm{P}=0.182$, respectively), significantly extended the OS (Fig. 5). The survival analysis also identified that the patient age and the time interval between primary disease and gastric metastasis did not prolong the survival of patients with gastric metastases (Table II; $\mathrm{P}=0.166$ and $\mathrm{P}=0.998$, respectively). Multivariate analysis indicated that any other metastasis exist prior to stomach was an independent indicator of poor OS ( $\mathrm{P}=0.011$; Table IV).

\section{Discussion}

Metastatic involvement of the stomach secondary to breast cancer is rare and its management varies significantly from that of a primary gastric cancer (7). A previous study reported a retrospective series of breast cancer metastases with an incidence of metastasis to the stomach of $0.1 \%$ (8). However, another study investigating the autopsies of 1,010 patients with cancer identified that gastric metastases were detected in 17 patients, yielding an incidence rate of $<1.7 \%$ (9). It was previously hypothesized that gastric metastases of breast cancer have been underestimated in the history of breast cancer diagnosis (10). This may occur primarily as a result of the diverse and nonspecific symptoms at clinical presentation, indistinguishable radiological and endoscopic features and the potentially lengthy disease-free survival period of patients with gastric metastases from breast cancer (11-13). Therefore, novel studies investigating the diagnosis and treatment received by patients with gastric metastasis from breast cancer are required to improve the therapeutic outcomes for this group. 
Of the patients with gastric metastases, the prevalent primary sites were breast cancer (27.9\%), followed by lung cancer (23.8\%), esophageal cancer (19.1\%), renal cell carcinoma (7.6\%) and malignant melanoma (7.0\%) (14). This may reflect the high incidence of breast cancer and lung cancer in the general population (11). Concordant with previous studies $(8,15,16)$, gastric metastases from breast cancer developed more frequently from ILC (65.4\%) compared with IDC (24.4\%; Table I). ILC comprises $\sim 15 \%$ of breast cancer cases and has clinical, biological and molecular distinctions compared with IDC (15). Borst et al (17) reported the metastatic patterns of ILC and IDC differ considerably. ILC has a greater propensity towards metastasizing to the gastrointestinal tract, gynaecological organs, peritoneum, retroperitoneum, adrenal glands and bone marrow $(17,18)$ and IDC frequently metastasizes to the lung, bones and liver (19). The factors that account for this variation in metastatic patterns are yet to be elucidated. A previous study hypothesized that the distinct ILC transcriptomic signature may be associated with metastatic behaviour (20). Fernandes et al (21) also reported that the absence of the cell-cell adhesion molecule E-CAD decreased the adhesiveness of tumor cells and may be responsible for the metastatic patterns of ILC. The incidence of ILC has been increasing over the previous two decades and this may occur as a result of the use of replacement hormonal therapy and improvements in diagnostic practice $(22,23)$. Therefore, the number of patients with gastric metastases that develop from breast cancer may also increase.

Due to nonspecific symptoms including dysphagia, dyspepsia, anorexia, abdominal pain, early satiety, nausea and vomiting and bleeding, the clinical presentation of gastric metastases from breast cancer mimics a primary gastric tumor (24). Concordant with previous studies, abdominal pain was the most common symptom in the current study (75.6\%; Table II). The pain associated with gastric metastasis is typically mild and localized to the epigastric region, which may mimic peptic ulcer disease and be relieved by eating. Furthermore, gastric metastases may develop several years following the initial diagnosis in patients with breast cancer (25). The median time between the diagnosis of primary breast cancer and gastric involvement was 60 months in the present study (Table II). Therefore, a patient with ILC that has been disease-free for several years may still be at risk of gastric metastases and exhibit gastrointestinal symptoms. Notably, gastric metastasis frequently occurs with synchronously with other sites of metastases $(11,24)$. In the current study, systemic metastases to other locations were detected in 27 patients prior to the diagnosis of gastric metastases (34.6\%). At the time of gastric metastasis diagnosis, 49 patients had concurrent metastases, which typically occurred in the bone (50\%), lung $(12.2 \%)$ and liver (20.4\%). Taken together, the present study emphasizes the importance of a comprehensive examination at the time of diagnosis of a patient's gastric metastasis, which may aid clinicians in providing an accurate diagnosis and an earlier initiation of effective treatment.

Breast cancer with stomach involvement presents a diagnostic challenge. Endoscopic investigation followed by a biopsy is the required procedure to provide a definitive diagnosis and histological comparisons between biopsy samples and the prior breast carcinoma specimens is recommended $(12,14)$.
Kim et al (11) had identified three main morphological features of gastric metastases in endoscopic examination as follows: Submucosal tumor masses with elevation and tip ulceration; non-ulcerative masses; multiple nodules of varying sizes. In the current study, gastric lesions were described as polypoid masses or volcano-like ulcers and mostly involved the lower third of the stomach, including the antrum and pylorus. However, gastric metastases that have spread throughout the mucosa and gastric wall may resemble primary gastric cancer, making clinical management problematic. Primary gastric diffuse signet-ring cell carcinoma and lobular breast carcinoma exhibit similar morphological features and these two neoplasms may be indistinguishable without further investigation (26). Furthermore, primary stomach cancer is also able to metastasize to the breast, which may complicate the diagnosis $(27,28)$. The use of a variety of methods, including immunochemistry, may be helpful to differentiate gastric metastasis with morphologically similar tumors and tumors of unknown origin. In the present study, the majority of the metastatic breast carcinomas were ER-, PR-, GCDFP-15 and CEA- positive and CK20-negative (Fig. 1) (29), whereas a primary stomach cancer is typically CK7- and CK20-positive and ER- and mammaglobin-negative (30). However, as an established mediator of the carcinogenic process, HER-2 is dysregulated in a number of types of solid tumor, including breast and stomach cancer (31) and therefore it may not be a suitable diagnostic marker for gastric metastasis from breast cancer. Taken together, it may be important for clinicians to not solely rely on the tumor biomarkers for the accurate diagnosis and treatment of gastric metastasis, due to the lack of a specific immunohistochemistry marker for breast cancer (32) and that the tumor phenotype may be unstable throughout the disease progression (33). Therefore, the diagnosis and appropriate treatment of gastric metastases of breast cancer may be improved using a diverse approach from the clinician along with the use of numerous diagnostic methods that aid pathological diagnosis.

To the best of our knowledge, a small number of studies have been reported to investigate the prognostic factors and standard treatment strategies for gastric metastases of breast cancer due to the low incidence rate. Certain previous studies advocated systemic therapy for the cases of breast cancer that have metastasized to the stomach, rather than using surgery as a primary treatment option $(12,34)$. Surgery may only be considered in cases of acute complications, including stomach bleeding, obstruction and perforation, to improve quality of life $(10,14)$. However, other studies also hypothesized that surgery may be the optimal first-line treatment for operable solitary breast cancer metastasis to the stomach $(29,35)$. As presented in Fig. 4A and B, survival analysis identified that surgical intervention and chemotherapy did not significantly extend $\mathrm{OS}(\mathrm{P}=0.134$ and $\mathrm{P}=0.182$, respectively), whereas hormonal therapy was demonstrated to be an effective strategy ( $\mathrm{P}=0.032$; Fig. 4). This was partly as the hormonal receptors are typically positive in patients with gastric metastases. As aforementioned, gastric metastases from breast cancer are more typical in ILC (Table I) and ILC tissues were significantly more likely to be ER and PR-positive $(18,36)$. Therefore, the present study hypothesizes that the biological features of the primary breast tumors may partly contribute to the sensitivity 
to hormonal therapy of gastric metastases. Concordant with previous studies $(24,37)$, the present study demonstrated that low HER-2 expression was identified in gastric lesions (6.4\%). Although trastuzumab (Herceptin) has been approved for the treatment of advanced metastatic gastric cancer with a positive HER-2 status, as defined by an immunohistochemistry $3+$ result (38), this group of patients were rarely treated with anti-HER2-targeted therapy. Further studies are important to evaluate the role of targeted therapy for this rare disease phenotype.

In conclusion, the present study indicated that systemic treatment strategies, including hormonal therapy, may be the optimal choice for gastric metastasis from breast cancer. The described case demonstrated that hormonal therapy was able to control the disease and provided a lengthy duration of relapse-free survival. Several limitations should be considered when interpreting the results of the present study. Firstly, the present study is retrospective and had a long duration and thus, the patients were heterogenous. In addition, the treatment modalities and the primary breast carcinoma features were heterogenous among distinct medical centres. In addition, the specific information concerning the primary tumor and follow-up was unavailable. The current study focuses on the contributing factors of survival and appropriate treatments for patients with breast cancer with gastric metastases. Prospective studies with a longer follow-up time and higher patient numbers may allow an improved understanding of the biological, pathological and clinicopathological characteristics, the clinical outcomes and the endoscopic features associated with gastric metastases from breast cancer.

\section{Acknowledgements}

This study was supported by The National Natural Science Foundation of China (grant nos. 81172522 and 81301858).

\section{References}

1. Siegel RL, Miller KD and Jemal A: Cancer statistics, 2015: CA Cancer J Clin 65: 5-29, 2015.

2. Wang $\mathrm{H}$, Zhang $\mathrm{C}$, Zhang J, Kong L, Zhu H and Yu J: The prognosis analysis of different metastasis pattern in patients with different breast cancer subtypes: A SEER based study. Oncotarget Dec 27, 2016 (Epub ahead of print).

3. Koike K, Kitahara K, Higaki M, Urata M, Yamazaki F and Noshiro H: Clinicopathological features of gastric metastasis from breast cancer in three cases. Breast Cancer 21: 629-634, 2014.

4. Cheng L and Li X: Breast imaging reporting and datasystem (BI-RADS) of magnetics resonance imaging: Breast mass. Gland Surg 1: 62-74, 2012.

5. Bast RC Jr, Klug TL, St John E, Jenison E, Niloff JM, Lazarus H, Berkowitz RS, Leavitt T, Griffiths CT, Parker L, et al: A radioimmunoassay using a monoclonal antibody to monitor the course of epithelial ovarian cancer. N Engl J Med 309: 883-887, 1983.

6. Singletary SE, Allred C, Ashley P, Bassett LW, Berry D, Bland KI, Borgen PI, Clark GM, Edge SB, Hayes DF, et al: Staging system for breast cancer: Revisions for the 6th edition of the AJCC cancer staging manual. Surg Clin North Am 83: 803-819, 2003

7. Almubarak MM, Laé M, Cacheux W, de Cremoux P, Pierga JY, Reyal F, Bennett SP, Falcou MC, Salmon RJ, Baranger B and Mariani P: Gastric metastasis of breast cancer: A single centre retrospective study. Dig Liver Dis 43: 823-827, 2011.

8. Abid A, Moffa C and Monga DK: Breast cancer metastasis to the GI tract may mimic primary gastric cancer. J Clin Oncol 31: e106-e107, 2013.
9. Taal BG, Boot $\mathrm{H}$, van Heerde $\mathrm{P}$, de Jong D, Hart AA and Burgers JM: Primary non-Hodgkin lymphoma of the stomach: Endoscopic pattern and prognosis in low versus high grade malignancy in relation to the MALT concept. Gut 39: 556-561, 1996.

10. Zelek L, Cottu PH, Mignot L, de Roquancourt A, Fizazi K, Cojean-Zelek I, Espie M and Marty M: Gastric metastases from breast cancer: A retrospective series of 12 patients. Am J Clin Oncol 24: 363-365, 2001.

11. Takeuchi H, Hiroshige S, Yoshikawa Y, Kusumoto T and Muto Y: A case of synchronous metastasis of breast cancer to stomach and colon. Anticancer Res 32: 4051-4055, 2012.

12. Taal BG, Peterse H and Boot H: Clinical presentation, endoscopic features and treatment of gastric metastases from breast carcinoma. Cancer 89: 2214-2221, 2000.

13. Kim GH, Ahn JY, Jung HY, Park YS, Kim MJ, Choi KD, Lee JH, Choi KS, Kim do H, Lim H, et al: Clinical and endoscopic features of metastatic tumors in the stomach. Gut Liver 9: 615-622, 2015.

14. Namikawa $T$ and Hanazaki K: Clinicopathological features and treatment outcomes of metastatic tumors in the stomach. Surg Today 44: 1392-1399, 2014.

15. Van Trappen P, Serreyn R, Elewaut AE, Cocquyt V and Van Belle S: Abdominal pain with anorexia in patients with breast carcinoma. Ann Oncol 9: 1243-1245, 1998.

16. Schwarz RE, Klimstra DS and Turnbull AD: Metastatic breast cancer masquerading as gastrointestinal primary. Am J Gastroenterol 93: 111-114, 1998.

17. Borst MJ and Ingold JA: Metastatic patterns of invasive lobular versus invasive ductal carcinoma of the breast. Surgery 114: 637-642, 1993

18. Arpino G, Bardou VJ, Clark GM and Elledge RM: Infiltrating lobular carcinoma of the breast: Tumor characteristics and clinical outcome. Breast Cancer Res 6: R149-R156, 2004.

19. Sobinsky JD, Willson TD, Podbielski FJ and Connolly MM: Unusual metastatic patterns of invasive lobular carcinoma of the breast. Case Rep Oncol Med 2013: 986517, 2013.

20. Gruel N, Lucchesi C, Raynal V, Rodrigues MJ, Pierron G, Goudefroye R, Cottu P, Reyal F, Sastre-Garau X, Fourquet A, et al: Lobular invasive carcinoma of the breast is a molecular entity distinct from luminal invasive ductal carcinoma. Eur J Cancer 46: 2399-2407, 2010

21. Fernandes GS, Corrêa TS, Carvalho EP, Katz A and Hoff PM: Gastric and endobronchial metastases in a case of lobular breast cancer. Case Rep Oncol 6: 555-560, 2013.

22. Arrangoiz R, Papavasiliou P, Dushkin H and Farma JM: Case report and literature review: Metastatic lobular carcinoma of the breast an unusual presentation. Int J Surg Case Rep 2: 301-305, 2011.

23. Bharat A, Gao F and Margenthaler JA: Tumor characteristics and patient outcomes are similar between invasive lobular and mixed invasive ductal/lobular breast cancers but differ from pure invasive ductal breast cancers. Am J Surg 198: 516-519, 2009.

24. Pectasides D, Psyrri A, Pliarchopoulou K, Floros T, Papaxoinis G, Skondra M, Papatsibas G, Macheras A, Athanasas G, Arapantoni-Datioti P and Economopoulos T: Gastric metastases originating from breast cancer: Report of 8 cases and review of the literature. Anticancer Res 29: 4759-4763, 2009.

25. Ricciuti B, Leonardi GC, Ravaioli N, De Giglio A, Brambilla M, Prosperi E, Ribacchi F, Meacci M, Crinò L, Maiettini D, et al: Ductal breast carcinoma metastatic to the stomach resembling primary linitis plastica in a male patient. J Breast Cancer 19: 324-329, 2016

26. Yoshida Y: Metastases and primary neoplasms of the stomach in patients with breast cancer. Am J Surg 125: 738-743, 1973.

27. Sataloff DM, Dentchev D, Henry DH and Weese JL: Isolated breast metastases from primary gastric adenocarcinoma. Breast J 6: 62, 2000 .

28. Sato T, Muto I, Fushiki M, Hasegawa M, Hasegawa M, Sakai T and Sekiya M: Metastatic breast cancer from gastric and ovarian cancer, mimicking inflammatory breast cancer: Report of two cases. Breast Cancer 15: 315-320, 2008.

29. Sarkut P, Ozer A, Gulcu B, Ozturk E, Gokgoz S and Ugras N: An extremely rare cause of gastric outlet: Breast lobular carcinoma metastases to stomach. Breast J 20: 312-313, 2014.

30. Tot T: The role of cytokeratins 20 and 7 and estrogen receptor analysis in separation of metastatic lobular carcinoma of the breast and metastatic signet ring cell carcinoma of the gastrointestinal tract. APMIS 108: 467-472, 2000.

31. Martin V, Cappuzzo F, Mazzucchelli L and Frattini M: HER2 in solid tumors: More than 10 years under the microscope; where are we now? Future Oncol 10: 1469-1486, 2014. 
32. Brown RW, Campagna LB, Dunn JK and Cagle PT: Immunohistochemical identification of tumor markers in metastatic adenocarcinoma. A diagnostic adjunct in the determination of primary site. Am J Clin Pathol 107: 12-19, 1997.

33. Guarneri V, Giovannelli S, Ficarra G, Bettelli S, Maiorana A, Piacentini F, Barbieri E, Dieci MV, D'Amico R, Jovic G and Conte P: Comparison of HER-2 and hormone receptor expression in primary breast cancers and asynchronous paired metastases: Impact on patient management. Oncologist 13: 838-844, 2008.

34. Dassen AE, Lips DJ, Hoekstra CJ, Pruijt JF and Bosscha K: FDG-PET has no definite role in preoperative imaging in gastric cancer. Eur J Surg Oncol 35: 449-455, 2009.

35. Gadde R, Tamariz L, Hanna M, Avisar E, Livingstone A, Franceschi D and Yakoub D: Metastatic gastric cancer (MGC) patients: Can we improve survival by metastasectomy? A systematic review and meta-analysis. J Surg Oncol 112: 38-45, 2015.
36. Wasif N, Maggard MA, Ko CY and Giuliano AE: Invasive lobular vs. ductal breast cancer: A stage-matched comparison of outcomes. Ann Surg Oncol 17: 1862-1869, 2010.

37. Ambroggi M, Stroppa EM, Mordenti P, Biasini C, Zangrandi A, Michieletti E, Belloni E and Cavanna L: Metastatic breast cancer to the gastrointestinal tract: Report of five cases and review of the literature. Int J Breast Cancer 2012: 439023, 2012.

38. Spackman E, Rice S, Norman G, Suh DC, Eastwood A and Palmer S: Trastuzumab for the treatment of HER2-positive metastatic gastric cancer: A NICE single technology appraisal. Pharmacoeconomics 31: 185-194, 2013. 